\title{
The Effectiveness of Cognitive Behavioral Therapy for Eating Disorders
}

\author{
Valbona Uka \\ Muhamet Karameta ${ }^{2}$ \\ 'Universi College, Faculty of Nursing, Prishtine, Kosovo \\ 2University Clinical Centre of Kosovo, Psychiatric Clinic, Prishtine, Kosovo
}

\begin{abstract}
Introduction: Considering the importance of studying young Kosovars, with a special focus on the mental disorders that are appearing every day in our society, we have researched the effectiveness of behavioral cognitive therapy in patients with eating disorders.
\end{abstract}

Purpose: To prove the effectiveness and impact that cognitive-behavioral therapy has on patients with eating disorders, compared to patients who use only medications.

Methodology: The research was conceptualized as a cause-and-effect experiment, lasted 8-12 weeks for 60 patients selected with eating disorders, in 30 patients CBT and medications were applied and in 30 patients only medications were used. In the same patients, it was measured by the level of anxiety and depression at the beginning in the middle and at the end of therapy.

Results: According to the results, there is a statistically significant difference in the level of anxiety in patients with bulimia nervosa who applied CBT compared with patients who used only medications. There is also a statistically significant difference in the level of depression in patients with anorexia nervosa who applied CBT compared with patients who used only medications. According to the data, the duration of the disease in patients with CBT lasts less than 6 months, while patients with medication last 8-12 months.

Conclusions: According to research facts, the application of behavioral cognitive therapy has shown high efficacy in the treatment of eating disorders, compared to patients who have used only medications. Applying cognitive behavioral therapy still remains a challenge for our society.

\section{Keywords}

mental health, cognitive behavioral therapy, anorexia nervosa, bulimia nervosa, patient, effective.

Address for correspondence:

Valbona Uka, Universi College, Faculty of Nursing, Prishtine, Kosovo,. e-mail: vuka5@live.com

This work is licensed under a Creative Commons Attribution-

Submitted for publication: 22 June 2020

NonCommercial 4.0 International License (CC BY-NC 4.0).

\section{(c) (1) (3)}

(C) Copyright: Uka, Karameta, 2020 


\section{Introduction}

Eating disorders are serious mental disorders that can affect people of any age, gender, race, ethnicity and socioeconomic group, but are treatable disorders. Eating disorders are very complex, and despite the scientific research to understand them, the biological basis, behavior and social aspect of these disorders remain to be explored in the future.

In post-war Kosovo, numerous efforts have been made regarding health reform, but these reforms have been mainly oriented towards general health services, and have not included psychological services at the appropriate level. Also in health services there is no distribution of psychologists in adequate places where they would have had the opportunity to provide psychological and psychotherapeutic services for mental disorders, where the number of young people with these disorders is increasing every day. Considering the importance of studying young Kosovars, with a special focus on the mental disorders that are appearing every day in our society, I have researched the effectiveness of behavioral cognitive therapy in patients with eating disorders.

\section{Literature review:}

When we talk about research on eating disorders, we know that it is a broad form of research in the world, but in our country at the moment it is not practiced enough. Many foreign authors have addressed this topic which has been of great importance as it is useful to diagnose eating disorders and its consequences on mental health in patients, as well as the importance of applying cognitive behavioral therapy to help patients in the treatment of eating disorders.

The literature review focuses on the identification of eating disorders and the effectiveness of the application of cognitive behavioral therapy to patients.

\section{Purpose}

The main purpose of this study is the effectiveness of behavioral cognitive therapy in patients with eating disorders. This study aims to identify the impact that behavioral cognitive therapy has on patients with anorexia nervosa and bulimia nervosa by analyzing the state and level of anxiety and depression in patients at the beginning of therapy and the probability of reducing anxiety and depression at the end of therapy.

\section{Research Hypotheses:}

$\mathbf{H 1}$ : There is a statistically significant difference in the level of anxiety in patients with bulimia nervosa who apply behavioral cognitive therapy compared to patients who do not apply behavioral cognitive therapy.

H2: There is a statistically significant difference in the level of depression in patients with anorexia nervosa who apply cognitive-behavioral therapy compared to patients who do not apply cognitive-behavioral therapy).

\section{Methodology}

The research was conceptualized as a causeexperiment, behavioral cognitive therapy was applied in the work environment where I work, and was the same for all subjects who participated in the study. The research was conceived as a cause-and-effect experiment, lasting 8-12 weeks for 60 selected patients with eating disorders, where in 30 patients $C B T$ and medication use was applied and in 30 patients only medications were used. In the same patients, anxiety and depression levels were measured at the beginning between and at the end of therapy. Since the character of the research is cause-experimental we can prove that through the intervention with cognitive behavioral therapy for a period of 3-5 months in the group of patients with anxiety and depression it is expected that their condition will improve.

Measuring techniques and instruments:

The measuring instruments in this research are; Demographic questionnaire; Anxiety Inventory (Beck); Depression Inventory (Beck); Application of cognitive behavioral therapy.

The application of behavioral cognitive therapy was based on the CBT cognitive model that focuses on thinking, beliefs, feelings, and behavior. The CBT model is developed through these phases: Stage one-starting well treatment planning and building therapeutic alliance; Stage two- treatment evaluation and progress. Stage 
three- ongoing assessment of body image concern, eating disorder patterns and behavior, meal planning, events and emotional regulation. Stage four - ending well, relapse prevention planning, continuation of care.

\section{Results}

Demographic characteristics female gender presents with $61.67 \%$, versus male with 38.33.The age group $20-25$ years presents with $38.4 \%$, the age group 16-18 years presents with $46.6 \%$ and the age group 25-30 years presents with $15 \%$. Patients in the urban area present with $68.5 \%$, while in the rural area with $31.5 \%$. Clinical characteristics of patients: patients with eating disorders reported that they received medical services in the following institutions: family medicine center with $22.4 \%$, psychiatric hospital with $27.6 \%$, and mental health center outpatient visits with 50\%. According to the results, the duration of the disease in patients with $C B T$ is reported to last up to 6 months, while in patients with medication lasts from 8-12 months. Based on the statistical results, the coefficient of significance is presented with $\mathrm{F}=1.064 \mathrm{sig}=$ $0.048(0.01<p<0.05)$ so it means that there is a statistically significant difference in the degree of anxiety in patients with bulimia nervosa compared to patients who use only drugs.

Also, there is a statistically significant difference in the degree of depression throughout the research, at each stage of treatment, and have shown positive results especially those patients who have undergone CBT compared to patients who have used only medication, and thus the two hypotheses that are submitted in the research have been verified.

\section{Limitations and Strengths of the study}

This study has some limitations such as we do not have data regarding the exact number of people with eating disorders, the small number of participants in the research, considerable is the fact that the truth of the answers, sometimes may have been influenced considering that the completion of the questionnaires, in some cases, took place in poor health. In terms of strengths, this study is one of the first studies in our country that applies behavioral cognitive therapy to patients with eating disorders.

\section{Conclusions and recommendations}

Behavioral cognitive therapy is one of the most researched and widely used approaches to psychotherapy in the world, and this is also confirmed by the large number of controlled clinical trials that have been conducted to date. Based on this research, we conclude that cognitive behavioral therapy is highly effective in patients with eating disorders by reducing depressive symptoms, and reducing anxiety levels compared to patients with eating disorders who have used only medication. Behavioral cognitive therapy in general is a reliable therapy for the treatment of eating disorders especially for the significant positive effects it manages to have especially in a relatively short period. Considering the effectiveness of cognitive behavioral therapy, it is recommended to apply CBT in health institutions, at primary and secondary level, which would help the mental health of the individual.

\section{Conflict of interests}

Authors declare that they have no conflict of interests.

\section{References}

American Psychiatric

Association,(2018).Diagnostic and statistical manual of Mental Disorders -DSM-V Washington. DC: Author

American Psychiatric Association. (2000). Practice guidelines for the treatment of

patients with eating disorders (revision). American Journal of Psychiatry, 157.

Beck.(1995)Cognitive Therapy: Basic and Beyond. New York. The Guilford Press.

Beck, Aster, R. \& Brown, G. (1996).The Beck Depression Inventory - Second Edition Manual. SanAntonio, TX: The Psychological Corporation.

Beck. S. Judith. (1995), Cognitive Behavior Therapy, New York. The Guilford Press.

Borkovec,T\& Ruscio,A.M.(2001).Psychotherapy for generalized anxiety disorder. Journal of Clinical Psychiatry, 62 (suppl. 11), 37-42. 
Dobson, K. (2010).Handbook of CognitiveBehavioral Therapies. New York. The Guilford Press.

Gilbert, Lehar. (2007).The therapeutic Relationship in the Cognitive Behavioral Psychotherapies. London. Sage.

Hendricks Vishay RC, Kinesis GPJ.(2009).Psychological treatments for bulimia nervosa and binning. Pub Med.
Padesky,C.(2011).Collaborative

Case Conceptualization Rating Scale, Coding Manual. New York. Guilford.

Fairburn, C. (2005). Evidence-based treatment of anorexia nervosa. International Journal of Eating Disorders, 37, S26-S30 